\title{
Venous pseudo-aneurysm as a late complication of short-term central venous catheterisation Heyman Luckraz*
}

\author{
Address: The Cardiac Trust Fund, Cardiac Centre, Pamplemousses, Mauritius \\ Email: Heyman Luckraz* - HeymanLuckraz@aol.com \\ * Corresponding author
}

This article is available from: http://www.cardiovascularultrasound.com/content/l///6

(C) 2003 Luckraz; licensee BioMed Central Ltd. This is an Open Access article: verbatim copying and redistribution of this article are permitted in all media for any purpose, provided this notice is preserved along with the article's original URL.

\begin{abstract}
Complications following central venous catheterisation abound in the medical literature. This reflects the under-estimated potential hazards associated with this procedure as well as an increase in the number of such procedures performed in high-risk patients. We report on a case where a venous pseudo-aneurysm developed four months after the removal of a short-term central venous catheter in a moderately high-risk patient.
\end{abstract}

\section{Introduction}

There has been a significant increase in the number of internal jugular veins (IJV), which are cannulated for the purposes of haemodynamic monitoring and resuscitation, haemodialysis and the administration of drugs and parenteral nutrition. This has been paralleled by an increase in the number of the various complications associated with this procedure.

The overall complication rate of IJV cannulation is reported to be up to $14 \%$ [1]. Complications can be grouped as either immediate or late, and either localized or distal to the puncture site. They are further classified as vascular, neurological, pulmonary or others. The venous vascular complications which have been reported thus far include arterio-venous fistula [2-4] and superior sagittal sinus thrombosis [5]. Venous pseudoaneurysm has not been reported yet. We describe a case where such a complication developed and outline the management strategies adopted.

\section{Case report}

A 42-year old male underwent aortic and mitral valve replacements with mechanical valves. As per the usual perioperative preparation, an $8 \mathrm{~mm}$ diameter, $20.3 \mathrm{~cm}$ long triple lumen (Certofix/Braun, Melsungen, Germany) central venous pressure monitoring line was inserted during the anaesthetic work-up. The anterior approach [6] through the apex of Sedillot triangle was used. However, insertion of the central line by the resident anaesthetic trainee was met with some difficulty. He managed to locate the vein by using the aspiration technique with a steel cannula, but failed to feed the guide wire appropriately on three occasions. Finally, the anaesthetic consultant positioned the CVP line by using a slightly more proximal point of entry.

In the immediate post-operative period, there was excessive mediastinal hemorrhage, which required surgical reexploration. Unfortunately, when the patient was being transferred from his bed to the operating table, the central venous line became dislodged. The IJV was re-cannulated via the same site without difficulty. Thereafter, the patient 


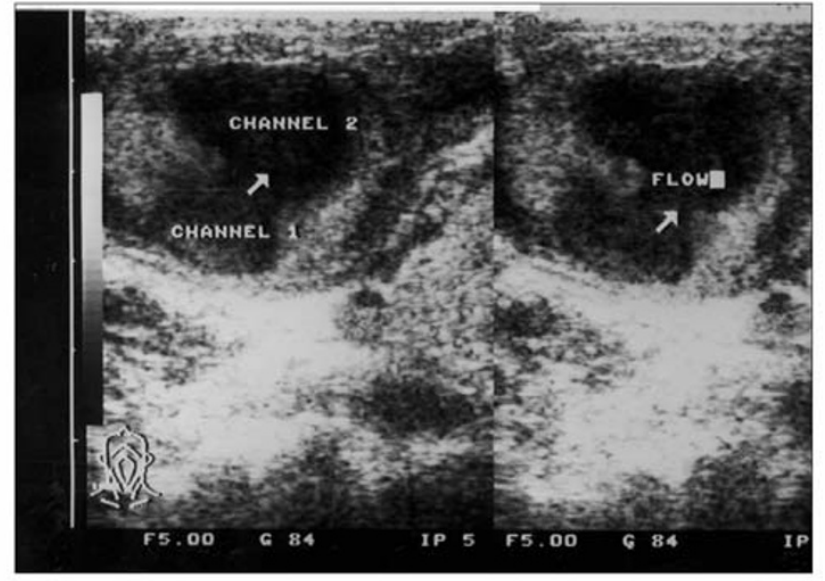

\section{Figure I}

Ultrasound scan of the right cervical region showing the right internal jugular vein (channel I) feeding the pseudoaneurysm (channel 2). had an uneventful post-operative recovery and the central line was removed on the second postoperative day. He was discharged from hospital after stabilisation of his INR (International Normalized Ratio) ten days postoperatively.

He was followed up regularly as an outpatient and his INR remained stable. Clinically he was asymptomatic. However, in the fourth post-operative month, he re-attended with a large lump over the area of the right sternocleidomastoid muscle. The lump, which had developed over a period of 24 hours after a bout of severe cough, was about $6 \mathrm{~cm}$ in diameter. It was non-tender, soft, mildly fluctuant and nontransilluminable.

There were three healed puncture-site scars representative of the previous different attempts at inserting the triple lumen catheter, on the skin over the lump. There was no bruit or thrill over the lump but a hum was audible. When the patient coughed, the lump increased in size momentarily and felt harder when the patient was asked to perform the Valsalva manoeuver. There was no dyspnoea, dysphagia or change in his voice.

His INR was 4.1 on $7.5 \mathrm{mg}$ of warfarin. Hemoglobin was 13.5 gdl- 1 and platelet count was $234 \times 10^{9} \mathrm{~L}^{-1}$. An infectious screen, including blood cultures, was negative.

An ultrasound scan was performed (Figure 1) and a $4 \mathrm{~cm}$ cystic lesion, separate from the right thyroid lobe, was identified. The cystic lesion was deep to the right sterno-

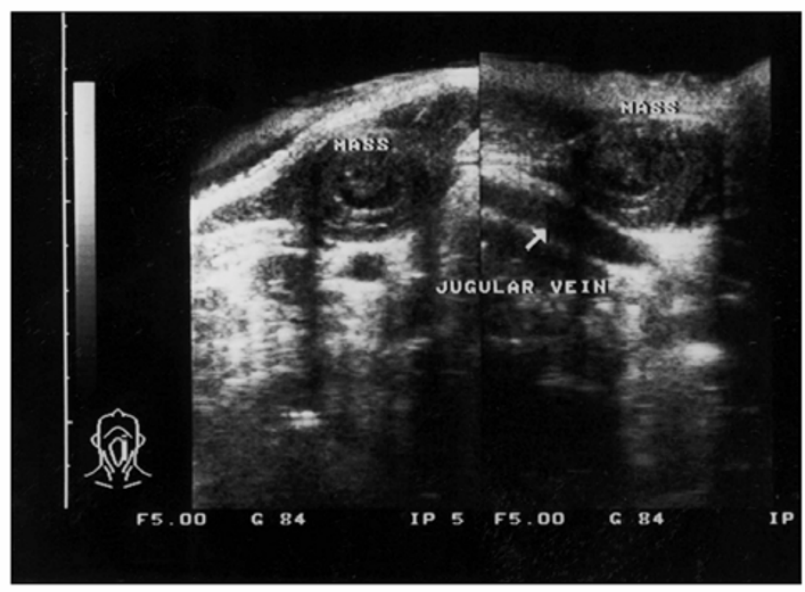

Figure 2

Ultrasound scan of the right internal jugular vein three months later demonstrating the closure of the feeding channel and shrinkage of the aneurysm. Clots are visible within the aneurysm.

mastoid muscle. There was a feeding channel from the underlying right internal jugular vein. CT scan with contrast confirmed the vascular nature of the cyst. The patient was kept under medical observation for a few days and there was no change in the size of the lump. The INR remained stable between 3.5 and 4.5. A conservative approach was adopted and was discharged from hospital within a week of admission. Three months later he remained stable. The lump had shrunk in size and the patient remained asymptomatic. His INR was maintained in the above range. A repeat ultrasound scan confirmed the closure of the feeding channel from the right IJV to the pseudo-aneurysm and appearance of clot within the latter (Figure 2).

\section{Discussion}

Central venous access is now commonly used peri-operatively as it not only provides a means of administering fluids and drugs, but also allows invasive monitoring of cardiac parameters. On a long-term basis its use is well recognized for long-term chemotherapy and parenteral feeding. However, complications rate of up to $14 \%$ [1] have been reported when central venous access is acquired.

Failure to cannulate the vein may be partly due to an aberrant anatomy of the internal jugular vein, which is estimated to be present in up to $8.5 \%$ of patients [7]. Patients who are either obese or who have short neck represent an additional challenge to such an extent that cannulation, then, becomes safer by using ultrasound guidance [8]. 
Vascular problems after IJV cannulation via the Seldinger technique [9] represent around $8 \%$ of the complications [10]. The bulk is related to carotid artery puncture [10,11], which may lead to neck haematoma and subsequent upper airways obstruction $[12,13]$. Venous complications are rare. Those reported so far, are either arterio-venous fistulae formation [2-4] or superior sagittal sinus thrombosis [5].

In this case report, the recurrent attempts at inserting the central line damaged to the wall of the right internal jugular vein. The end result was a weakened venous wall and most probably, the formation of a clinically non-significant venous pseudo-aneurysm. There was no or minimal localized thrombus as the patient was fully anti-coagulated. Following the severe bout of coughing, there was a significant increase in the venous pressure causing ballooning of the venous pseudo-aneurysm. The connective tissue sheath of the surrounding structures limited the size of the lesion. The size was further limited by the blood which collected within the aneurysmal sac creating a tamponade effect on the leak from the feeding vein. The tamponade was effective because the low pressure that prevails in the IJV could not maintain the blood flow from the IJV to the pseudo-aneurysm. The venous wall was, then, strengthened when the haematoma in the sac became organized clot. Thus, the feeding channel was closed and the lump shrunk in size.

This clinical complication was managed conservatively with favorable outcome. If the lump did not resolve, enlarged, or if the patient developed symptoms of space occupying lesions such as dyspnoea and dysphagia, then a surgical approach to close the feeding channel, aneurysmectomy and IJV repair would have been necessary. However, there is always the risk that either clot may embolise from the pseudo-aneurysm or there may even be a propagated thrombus formation which would lead to IJV obstruction and impairment of the venous drainage from the head and neck region. Surgical drainage of chronic haematoma following IJV cannulation has been previously described by Brown \& Wallace [14]. Non-surgical treatment was adequate in this case and successful closure of the venous pseudoaneurysm was achieved.

This complication was unexpected, as the central venous catheter was in-situ for only a short period of time. Unfortunately the multiple attempts at IJV catheterization prior to the valve replacement surgery along with its re-insertion for the re-exploration procedure weakened the venous wall and predisposed this anti-coagulated patient to this complication. It seems fair to conclude that in highrisk patients (as in this case with planned post-operative anticoagulation), multiple attempts at puncturing the same central vein should be avoided and alternative routes should be used if one attempt fails. Moreover, the use of ultrasound to locate the vein prior to catheterization will reduce the need for multiple blind attempts $[8,10]$.

\section{Conclusion}

Central venous cannulation has a significant complication rate. In high-risk patients, the procedure is best performed by experienced hands rather than the trainee staff and preferably under ultrasound guidance. Venous pseudo-aneurysm can be managed conservatively in the absence of complicating features.

\section{References}

I. Eisenhauer ED, Derveloy RF and Hastings PR Prospective evaluation of central venous pressure (CVP) catheters in a large city-county hospital Annals of Surgery 1982, 196:560-4

2. Stock $U$, Link J and Dutschke $P$ latrogenic vertebrojugular arteriovenous fistula Anaesthesia 1996, 5 I:687-88

3. Lambrinidis M, Vasdev A, Boubagra K, Bessou P and Crouzet G Traitement endovasculaire d'une fistule arterioveineuse ioatrogene. A propos d'un cas Journal de Radiologie 1992, 73:691-3

4. Danenberg HD, Hasin Y, Milgalter E, Gilon D, Zelinger J, Castaing D, Azoulay D, Putterman C, Bismuth H, Samuel D and Shouval D Aorto-atrial fistula following internal jugular vein catheterization European Heart Journal 1995, 16:279-81

5. Stephens PH, Lennox G, Hirsch N and Miller D Superior sagittal sinus thrombosis after internal jugular vein cannulation $\mathrm{Br} J$ Anaesth 1991, 67:476-9

6. Defalque RJ Percutaneous catheterization of the internal jugular vein Anaesthesia and Analgesia 1974, 53:1 I6-2 I

7. Denys BG and Uretsky BF Anatomical variations of internal jugular vein location: Impact on central venous access Crit Care Med 1991, 19:1516-9

8. Gallieni M and Cozzolino M Uncomplicated central vein catheterization of high-risk patients with real time ultrasound guidance Int J Artif Organs 1995, 18: I 17-21

9. Seldinger SI Catheter replacement of the needle in percutaneous arteriography Acta Radiol 1953, 39:368-76

10. Conz PA, Dissegna D, Rodighiero MP and La Greca G Cannulation of the internal jugular vein: comparison of the classic Seldinger technique and an ultrasound guided method J Nephrol 1997, 10:3II-3

II. Chudhari LS, Karmarkar US, Dixit RT and Sonia K Comparison of two different approaches for internal jugular vein cannulation in surgical patients J Postgrad Med 1998, 44:57-62

12. Randalls B and Toomey PJ Laryngeal oedema from a neck hematoma. A complication of internal jugular vein cannulation Anaesthesia 1990, 45:850-2

13. Lo WK and Chong JL Neck haematoma and airway obstruction in a pre-eclamptic patient: a complication of internal jugular vein cannulation Anaesth Intens Care 1997, 25:423-5

14. Brown CS and Wallace CT Chronic hematoma: a complication of percutaneous catheterization of the internal jugular vein Anesthesiology 1976, 45:368-9 